\title{
Percutaneous Irreversible Electroporation as First-line Treatment of Locally Advanced Pancreatic Cancer
}

\author{
CHRISTOPHER MÅNSSON ${ }^{1}$, RICHARD BRAHMSTAEDT $^{1}$, PETER NYGREN ${ }^{2}$, \\ ANDERS NILSSON $^{1}$, JOZEF URDZIK ${ }^{1}$ and BRITT-MARIE KARLSON ${ }^{1}$ \\ ${ }^{I}$ Department of Surgical Sciences, Uppsala University, Uppsala, Sweden; \\ ${ }^{2}$ Department of Immunology, Genetics and Pathology, Uppsala University, Uppsala, Sweden
}

\begin{abstract}
Background/Aim: Irreversible electroporation (IRE) has recently been used as an experimental ablation treatment following systemic chemotherapy in locally advanced pancreatic cancer (LAPC). The primary aim of this study was to evaluate survival of LAPC patients after IRE prior to chemotherapy. The secondary aim was to examine the complication rates. Patients and Methods: Twenty-four patients with LAPC were included and treated with percutaneous ultrasound-guided IRE under general anesthesia. Survival data from the National Quality Registry for Pancreatic and Periampullary Cancer for LAPC during the same period were used for comparison. Results: The median survival after diagnosis was 13.3 months in the IRE group compared to 9.9 months in the registry group $(p=0.511)$. Six patients had a severe complication after IRE treatment. Conclusion: No obvious gain in survival was observed with IRE as the first line treatment of LAPC and IRE was associated with severe complications. This study does not support percutaneous IRE in this setting.
\end{abstract}

Locally advanced pancreatic cancer (LAPC) has a poor prognosis even with optimal standard treatment and it is the fourth leading cause of cancer-related deaths in the western world (1). The poor prognosis has led to several trials investigating new treatment modalities, including radiofrequency, microwave and cryoablation. Photodynamic therapy and high-intensity focused ultrasound have also been tried with minor improvements to the prognosis (2). In recent years, several studies have been published about the use of irreversible electroporation (IRE) in LAPC, mostly following

Correspondence to: Christopher Månsson, Department of Surgical Sciences, Uppsala University, 75185 Uppsala, Sweden. Tel: +46 186114505, Fax: +46 186114508, e-mail: christopher.mansson@ surgsci.uu.se

Key Words: Pancreatic neoplasms, electroporation, interventional ultrasonography. treatment with chemotherapy $(1,3-5)$. However, Belfiore et $a l$. have presented data on IRE followed by chemotherapy in 20 patients with LAPC and were able to perform $\mathrm{R}_{0}$ resections on three patients (6). Our study represents our experience with IRE prior to chemotherapy in LAPC patients. The primary aim was to investigate overall survival and the secondary aim was to examine the rate of complications in this setting.

\section{Patients and Methods}

Patients. Patients included in our study had biopsy-proven LAPC defined as superior mesenteric- or celiac-artery encasement, aortic invasion or unreconstructable superior mesenteric- or portal-vein involvement, with no evidence of metastatic disease on abdominal and thoracic computed tomography (7) or at laparotomy, and no prior systemic treatment. Exclusion criteria were age $<18$, implanted electronic devices, ASA score IV, expected survival $<3$ months, pregnancy, epilepsy, severe heart disease, and tumor diameter $>5 \mathrm{~cm}$. All patients signed a consent form before treatment. Prior to inclusion, all patients were discussed at a multi-disciplinary team conference. Following IRE, starting adjuvant chemotherapy was recommended.

Twenty-five patients were considered a sufficient number to allow for preliminary conclusions on survival and complications. In the case of computed tomography $(\mathrm{CT})$ evaluated response to the IRE treatment, indicating that $\mathrm{R}_{0}$ resection could be achieved, resection would be scheduled. Chemotherapy was to be started following local recurrence. The oncologist in charge of the patient decided on chemotherapy after IRE. For comparison, we used survival data from the National Quality Registry for Pancreatic and Periampullary Cancer for patients with LAPC diagnosed during our inclusion period between 2013 and 2015 and scheduled for active, but not curative, treatment. We included LAPC cases diagnosed both with radiology and during laparotomy with curative intent. This group also contained our study patients. One difference compared with our previous study (5) is that the date of diagnosis is the date of radiology and not the date of the biopsy, because, to comply with the principles of the National Quality Registry for Pancreatic and Periampullary Cancer, we wanted the groups to be as similar as possible. It was not possible to extract co-morbidities from the National Quality Registry for Pancreatic and Periampullary Cancer and the groups were not be matched. The coverage of the National Quality Registry for Pancreatic and Periampullary Cancer during the study period was well over $90 \%$ (8). 
Twenty-four patients, 15 men and 9 women with a median age at diagnosis of 68.0 years, were included. After 24 patients were treated, we encountered difficulties with our ability to provide IRE within a reasonable time from the pancreatic cancer diagnosis and the study was stopped for ethical reasons, since this caused a delay in the standard of care, i.e. chemotherapy. All patients who were scheduled for IRE at the multi-disciplinary team conference received it. Of the 24 patients, eight had gone through a laparotomy with curative intent before inclusion but were found to have LAPC. Computed tomography confirmed that the rest had LAPC. Median tumor diameter was $30 \mathrm{~mm}$ and 18 of the tumors were located in the head of the pancreas and 6 in the body. The median time between the date of diagnosis and IRE treatment was 89 days. Eighteen of the patients had an ASA score of 2, and six had an ASA score of 3 (Table I). Data from 299 patients with LAPC, 149 men and 150 women, with a median age at diagnosis 70 years, were retrieved from the National Quality Registry for Pancreatic and Periampullary and were used as a control. Thirty-nine of them were diagnosed at laparotomy.

IRE procedure. The IRE treatment used was identical to that in our previous study (5). Briefly, the equipment from Angiodynamics System (Queensbury, NY, USA) known as Nanoknife was used. An ultrasound was conducted the day before the treatment to rule out metastases and to plan the needle placement. All needles were placed percutaneously trans-abdominally using ultrasound guidance. The active length of the needles was $15 \mathrm{~mm}$ and the needles were placed parallel to each other. Four to six needles were used for each patient. When the tumor's diameter exceeded $2 \mathrm{~cm}$, a needle was also placed in the center of the tumor. Initially, a test with 10 pulses of $1,200 \mathrm{~V} / \mathrm{cm}$ was sent and then the current was adjusted up or down to achieve an end current of 30-50 ampere. However, it was not possible to exceed $1,500 \mathrm{~V} / \mathrm{cm}$. After the 10 test pulses were administered, another 90 pulses were given as treatment. A pullback technique was used in all patients to cover the entire tumor and in five patients two pullbacks were used. IRE treatment was conducted under general anesthesia with deep neuromuscular block.

Post-IRE surveillance and follow-up. After IRE the patients were observed in-hospital for at least 3 days and an ultrasound was conducted to rule out complications before discharge. We defined a severe complication as Clavien-Dindo $>2$ that presented itself within 30 days of treatment (9). Another ultrasound and clinical assessment were performed after one month along with an assessment of complications and signs of active tumor. If no signs of complications were seen, the patient was referred for post-IRE chemotherapy. Patients were then followed with contrast-enhanced ultrasound and CT every third month until disease recurrence or progression was seen. Since the groups were not matched regarding age and gender, a Cox regression was used to compare survival between our study group and the registry group. A $p$-value $<0.05$ was considered as significant.

The study was approved by the Uppsala Regional Ethics Committee, Uppsala, Sweden (Dnr 2013/254).

\section{Results}

Treatment. The median stay at the hospital after the treatment was 5 days. We had planned to keep the patients for at least 3 days for observation. However, two were deemed fit to go home after two days. Postoperative ultrasounds showed a complete ablation zone without signs of tumor in all but one patient. This patient had a small rim on the border of the ablation zone that showed washout of the contrast, and the ablation might not have been radical. One patient had signs of minor bleeding which had no clinical implications.

There was no response to the IRE ablation indicating that a $R_{0}$ resection could be achieved, and thus no attempts to resect were made. Three patients had no adjuvant chemotherapy after the IRE treatment, one due to rapidly progressive disease. The other two did not recover sufficiently after their complications. It was not possible to obtain data from the patients' charts as to how many cycles of chemotherapy they received. In the control group, only 77 of the 299 patient reports showed whether or not they received any radio- or chemotherapy. Of these 77 , four had no radioor chemotherapy, four had primary radiochemotherapy, 72 had chemotherapy, and four had radiotherapy.

Disease recurrence and survival. Recurrent disease was seen in 18 patients in the IRE group. Seven had a local recurrence, 10 had metastases and one patient had both. Median progression-free survival for those with recurrent disease was 3.9 months. The median time to local recurrence and metastases was 5.8 and 2.3 months, respectively.

Median survival after the IRE treatment was 13.3 months and at the end of the study only one patient was alive, albeit with known local recurrent disease. Median survival after diagnosis of LAPC in the patients in the National Quality Registry for Pancreatic and Periampullary Cancer was 9.9 months. Median survival did not differ statistically significantly between the groups $(\mathrm{p}=0.511)$ and the patients in the control group were significantly older $(\mathrm{p}=0.01)$. Survival is shown in the Kaplan-Meier curve in Figure 1.

Safety. Six patients had a severe complication, i.e. ClavienDindo $>2$, that presented itself within 30 days of treatment. One patient died from the treatment after 50 days and had a Clavien-Dindo grade of 5 . This was a case with a small duodenal perforation that led to infectious complications with an abscess and later with air in the portal vein in the liver that was treated with a covered stent in the portal vein. This patient did not completely recover and died 50 days after IRE.

Three patients had a $3 \mathrm{~b}$ complication that required laparotomy. The first patient had a laparotomy two days post IRE for a common bile duct perforation that was treated with a T-drain. The second case had also bile leakage with bilirubin in a drain, but at laparotomy no perforation was found. However, part of the gastric wall was inflamed and invaginated. The third patient had a laparotomy due to free air discovered on computer tomography. At the laparotomy, bile-stained fluid was found, but no perforation. A T-drain was put in place and 
Table I. Study data for the patients in the IRE group.

\begin{tabular}{|c|c|c|}
\hline & $\mathrm{N}$ or median & $\%$ or Q1-Q3 \\
\hline \multicolumn{3}{|l|}{ Gender } \\
\hline Male & 15 & 63 \\
\hline Female & 9 & 38 \\
\hline \multicolumn{3}{|l|}{ Age } \\
\hline (Years) & 68.0 & $(60.5-74.5)$ \\
\hline \multicolumn{3}{|l|}{ ASA } \\
\hline 2 & 18 & 75 \\
\hline 3 & 6 & 25 \\
\hline \multicolumn{3}{|c|}{ Surgical exploration before IRE } \\
\hline Yes & 8 & 33 \\
\hline No & 16 & 67 \\
\hline \multicolumn{2}{|c|}{ Time from diagnosis to treatment } & $(62-118)$ \\
\hline \multicolumn{3}{|c|}{ Location of tumor } \\
\hline Caput & 18 & 75 \\
\hline Corpus & 6 & 25 \\
\hline $\begin{array}{l}\text { Tumor maxim } \\
(\mathrm{mm})\end{array}$ & Tumor maximal diameter & $(30-40)$ \\
\hline \multicolumn{3}{|c|}{ Clavien-Dindo score } \\
\hline none or $\leq 2$ & 18 & 75 \\
\hline $3 \mathrm{a}$ & 2 & 8 \\
\hline $3 b$ & 3 & 13 \\
\hline 5 & 1 & 4 \\
\hline \multicolumn{3}{|l|}{ Hospital stay } \\
\hline (days) & 6.0 & $(4.0-12.5)$ \\
\hline \multicolumn{3}{|c|}{ Adjuvant chemotherapy } \\
\hline Yes & 21 & 88 \\
\hline No & 3 & 13 \\
\hline \multicolumn{3}{|c|}{ Survival after diagnosis } \\
\hline 3 months & & 96 \\
\hline 12 months & & 71 \\
\hline 24 months & & 8 \\
\hline \multicolumn{3}{|c|}{ Survival after treatment } \\
\hline 3 months & & 96 \\
\hline 12 months & & 42 \\
\hline 24 months & & 4 \\
\hline
\end{tabular}

pyloric exclusion with gastroenteroanastomosis was conducted.

The remaining two complications were Clavien-Dindo 3a. The first was an infection that was complicated by an ulcer that needed an endoscopy for treatment and the other was an amylase-containing abscess that was successfully treated with a percutaneous drain placed by ultrasound. There were also three cases with Grade 2 complications: one infection, one pancreatitis and one patient developed severe diarrhoea that was partially alleviated with pancreatic enzymes. An angiogram was conducted which showed an intact superior mesenteric artery and the diarrhoea was deemed to be due to mesenteric denervation.
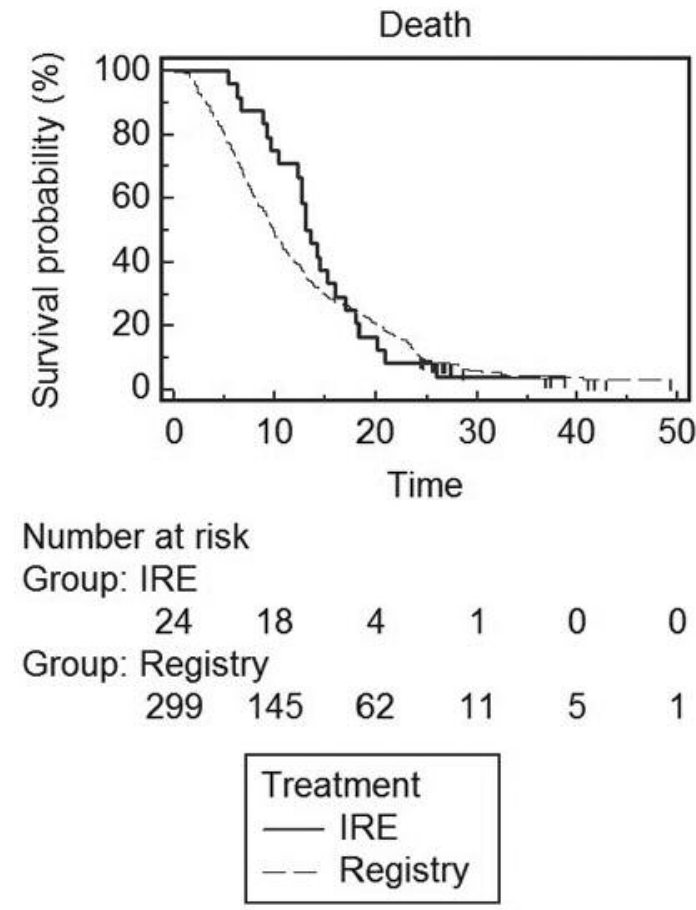

Figure 1. Kaplan-Meier curve of survival between the IRE group and the registry group.

\section{Discussion}

We observed no obvious gain in survival for the IRE group compared with register control patients and several IRE patients had severe complications. Belfiore et al. used a study design similar to ours and reported no severe complications (6). After the treatment, they were able to conduct a $R_{0}$ resection in three out of 20 patients. We had no cases in our study group that were deemed resectable after the treatment. However, the Kaplan-Meier analysis of survival in Belfiore's study showed a similar mean survival of 13.0 months (6). In their follow-up article with another nine patients, survival was 14.0 months (10). In that study, they compared their results with those in the literature and found an increased survival after IRE treatment. An interesting difference in Belfiore's studies is that they performed CT on postoperative day 1 and if no complication was found, adjuvant chemotherapy was started on the same day.

Compared to several other studies on IRE, this one has a control group. Although not matched for comorbidities, it gives us the survival for LAPC patients scheduled for active treatment in Sweden during the study period.

There are several limitations to this study. The most obvious is that it is non-randomized and the groups under comparison are not matched. We had several exclusion criteria in our study group (implanted electronic devices, ASA 
score IV, epilepsy, severe heart disease, and tumor diameter $>5.0 \mathrm{~cm}$ ) that were not excluded in the control group because they were not reported in the register. This might have affected the survival of the control group negatively.

The National Quality Registry for Pancreatic and Periampullary Cancer is not complete with respect to radiotherapy, radiochemotherapy and chemotherapy. This probably produces bias and we have performed no further analysis of the patients in the control group who received active treatment. There was a delay of 88 days between the date of the radiological diagnosis and the IRE treatment, due both to referral time and the waiting time at our center. Because of this delay, patients with rapidly progressive disease might never have been referred to us and they may be in the control group. This could lead to a positive selection for the IRE group.

The relatively long waiting time also raises some ethical issues since obviously some of the patients in the control group had to wait several months before any active treatment was started. Should IRE be used in this setting, the waiting time will have to be shorter.

The complication rate was higher than in our previous study with IRE after chemotherapy: in the present study, $25 \%$ of the patients had severe complications compared to $12.5 \%$ in the previous one (5). This was despite the use of identical techniques and with the same interventional radiologist doing the procedures. The only difference is in the timing of chemotherapy, the importance of which is difficult to judge. A larger study is needed to further evaluate the true complication rate after ultrasound-guided percutaneous IRE treatment.

The reason for the modest survival in the IRE group is probably that many of the patients had early undetectable metastatic disease and, thus, were not suitable for IRE. An induction period with chemotherapy is probably needed as a way to select patients more suitable for local treatment.

\section{Conclusion}

There is no obvious gain in survival from upfront IRE in LAPC cases and IRE in this setting is associated with several severe complications. This study does not support IRE in this setting.

\section{Conflicts of Interest}

Anders Nilsson has received travel funding from Angiodynamics for lectures.

\section{Authors' Contributions}

Study design: CM, RB, PN, AN, JU, BM; Data collection: CM, $\mathrm{BM}$; Data analysis and interpretation: CM, PN, JU, AN, BM; Drafting of the manuscript: CM, PN, BM; Critical revision and final approval: CM, RB, PN, AN, JU, BM.

\section{Acknowledgements}

The Authors would like to thank Maria Grünewald for her help with statistics and Mandy Trickett with language editing.

\section{References}

1 Paiella S, Butturini G, Frigerio I, Salvia R, Armatura G, Bacchion M, Fontana M, D'Onofrio M, Martone E and Bassi C: Safety and feasibility of Irreversible Electroporation (IRE) in patients with locally advanced pancreatic cancer: Results of a prospective study. Dig Surg 32(2): 90-97, 2015. PMID: 25765775. DOI: $10.1159 / 000375323$

2 Keane MG, Bramis K, Pereira SP and Fusai GK: Systematic review of novel ablative methods in locally advanced pancreatic cancer. World J Gastroenterol 20(9): 2267-2278, 2014. PMID: 24605026. DOI: $10.3748 / w j g . v 20.19 .2267$

3 Martin RC, 2nd, McFarland K, Ellis S and Velanovich V: Irreversible electroporation in locally advanced pancreatic cancer: potential improved overall survival. Ann Surg Oncol 20(3): S4439, 2013. PMID: 23128941. DOI: 10.1245/s10434-012-2736-1

4 Venkat S, Hosein PJ and Narayanan G: Percutaneous approach to irreversible electroporation of the pancreas: Miami Protocol. Techniques in vascular and interventional radiology 18(3): 153158, 2015. PMID: 26365545. DOI: 10.1053/j.tvir.2015.06.006

5 Mansson C, Brahmstaedt R, Nilsson A, Nygren P and Karlson BM: Percutaneous irreversible electroporation for treatment of locally advanced pancreatic cancer following chemotherapy or radiochemotherapy. Eur J Surg Oncol 42(9): 1401-1406, 2016. PMID: 26906114. DOI: 10.1016/j.ejso.2016.01.024

6 Belfiore MP, Ronza FM, Romano F, Ianniello GP, De Lucia G, Gallo C, Marsicano C, Di Gennaro TL and Belfiore G: Percutaneous CT-guided irreversible electroporation followed by chemotherapy as a novel neoadjuvant protocol in locally advanced pancreatic cancer: Our preliminary experience. Int J Surg 21(1): S34-39, 2015. PMID: 26118600. DOI: 10.1016/ j.jjsu.2015.06.049

7 Callery MP, Chang KJ, Fishman EK, Talamonti MS, William Traverso L and Linehan DC: Pretreatment assessment of resectable and borderline resectable pancreatic cancer: expert consensus statement. Ann Surg Oncol 16(7): 1727-1733, 2009. PMID: 19396496. DOI: 10.1245/s10434-009-0408-6

8 Styrgruppen. Kvalitetsregistret för tumörer i pankreas och periampulärt - Årsrapport 2016 [Available from: https://www.cancer centrum.se/globalassets/cancerdiagnoser/bukspottkortel/kvalitetsregi ster/arsrapport-pankreas-2016-for-publicering.pdf.

9 Dindo D, Demartines $\mathrm{N}$ and Clavien PA: Classification of surgical complications: a new proposal with evaluation in a cohort of 6336 patients and results of a survey. Ann Surg 240(2): 205-213, 2004. PMID: 15273542.

10 Belfiore G, Belfiore MP, Reginelli A, Capasso R, Romano F, Ianniello GP, Cappabianca S and Brunese L: Concurrent chemotherapy alone versus irreversible electroporation followed by chemotherapy on survival in patients with locally advanced pancreatic cancer. Med Oncol 34(3): 38, 2017. PMID: 28161827. DOI: $10.1007 / \mathrm{s} 12032-017-0887-4$

Received March 23, 2019 Revised April 10, 2019 Accepted April 11, 2019 